\title{
Redescription of Deutonymph of Curteria curticristata (Willmann, 1951) (Acari: Erythraeidae)
}

\author{
İbrahim KARAKURT* \\ Department of Home Care, Vocational School of Health Services, Erzincan Binali Yıldırım University, \\ Erzincan, Turkey
}

Geliş / Received: 02/09/2021, Kabul / Accepted: 01/12/2021

\begin{abstract}
Deutonymph of Curteria curticristata (Willmann, 1951) is redescribed based on single specimen which is found in Bayburt, Turkey. New morphometric data of this species are also presented in the study. C. curticristata is for the first time reported from Turkey with this study.
\end{abstract}

Keywords: Prostigmata, deutonymph, habitat, Turkey

Curteria curticristata (Willmann, 1951) (Acari: Erythraeidae) Deutonymphinin Yeniden Tanımlanması

\section{$\ddot{O} \mathbf{z}$}

Curteria curticristata (Willmann, 1951) deutonymphi, Türkiye'nin Bayburt ilinde bulunan tek bir örneğe dayandırılarak, yeniden tanımlandı. Ayrıca, çalışmada bu türe ait yeni morfometrik bilgiler sunuldu. $C$. curticristata bu çalışmayla, Türkiye'den ilk kez rapor edildi.

Anahtar Kelimeler: Prostigmata, deutonymph, habitat, Türkiye

\section{Introduction}

The genus Curteria was founded by Southcott (1961) with the type species Morieria curticristata Willmann, 1951. The genus Curteria currently represented by seven species; 2 of them are known from both active postlarval and larval stage while of which 2 only larvae and 3 from only active postlarval forms are known (Mąkol and Wohltmann, 2012). A comparative taxonomic analysis of active postlarval species of Curteria was provided by Gabryś (1992). Woltmann et al. (2007) described the larval forms of $C$. episcopalis (Koch, 1837) and $C$. southcotti Gabryś, 1992 previously known only from their active postlarval forms. Until now, only one larval Curteria species, C. duzgunesae (syn. Zhangiella duzgunesae) was reported from Turkey (Ankara province) by Saboori et al. (2007). Adult form of C. curticristata (syn. Morieria curticristata) was first described by Willmann (1951) from Austria and characterized as a species with a "high alpine" distribution by the same author. A deutonymph of C. curticristata (syn. Morieria curticristata) was recorded by Schweizer (1951) from Switzerland, despite doubts for Willmann as to whether these specimens are conspecific. However, Gabryś (1992) stated that the species given by Willmann and Schweizer are the same species, both as a result of his examinations and based on the relevant notes of Schweizer and Bader (1963). 
This study includes redescription of deutonymph of $C$. curticristata based on single specimen. Also, new morphometric data for this species are given.

\section{Material and methods}

Mite specimen was obtained in Bayburt Province, Turkey $\left(40^{\circ} 26^{\prime} \mathrm{N}, 40^{\circ} 07^{\prime} \mathrm{E}\right.$, Aydintepe plateau, $2230 \mathrm{~m}$, at a semi-aquatic grassland and mossy area, 17 May 2013, during the research process, it was not necessary to obtain permission for the collection of samples). Only one deutonymph was extracted in Berlese funnels and preserved in $70 \%$ ethyl alcohol. Specimen was fixed on slide in Hoyer's medium (Walter and Krantz, 2009) for microscopic studies. Leica DM 4000 microscope was used for measurements, photographs and drawings. The morphological terminology follows Gabryś (1992). All measurements are given in micrometers $(\mu \mathrm{m})$.

Voucher specimen was deposited in Acarology Laboratory of Erzincan Binali Y1ldırım University, Turkey (EBYU).

\section{Results}

Family Erythraeidae Robineau-Desvoidy, 1828

Genus Curteria Southcott, 1961

Type species Morieria curticristata Willmann, 1951

Diagnosis. Adult and deutonymph: see Woltmann et al. (2007).

Larva. see Woltmann et al. (2007).

\section{Curteria curticristata (Willmann, 1951)}

Diagnosis. Deutonymph. Palp tibia and genu without conalae. Crista metopica consists of anterior and posterior sensillary areas and a straight rod extending between them. Dorsal setae spiniform, almost smooth and short $(<60)$. Stems are slightly narrowed terminally and covered sparsely with delicate barbs. L/W of tarsus I $<2,5$.

Larva. Not known. 


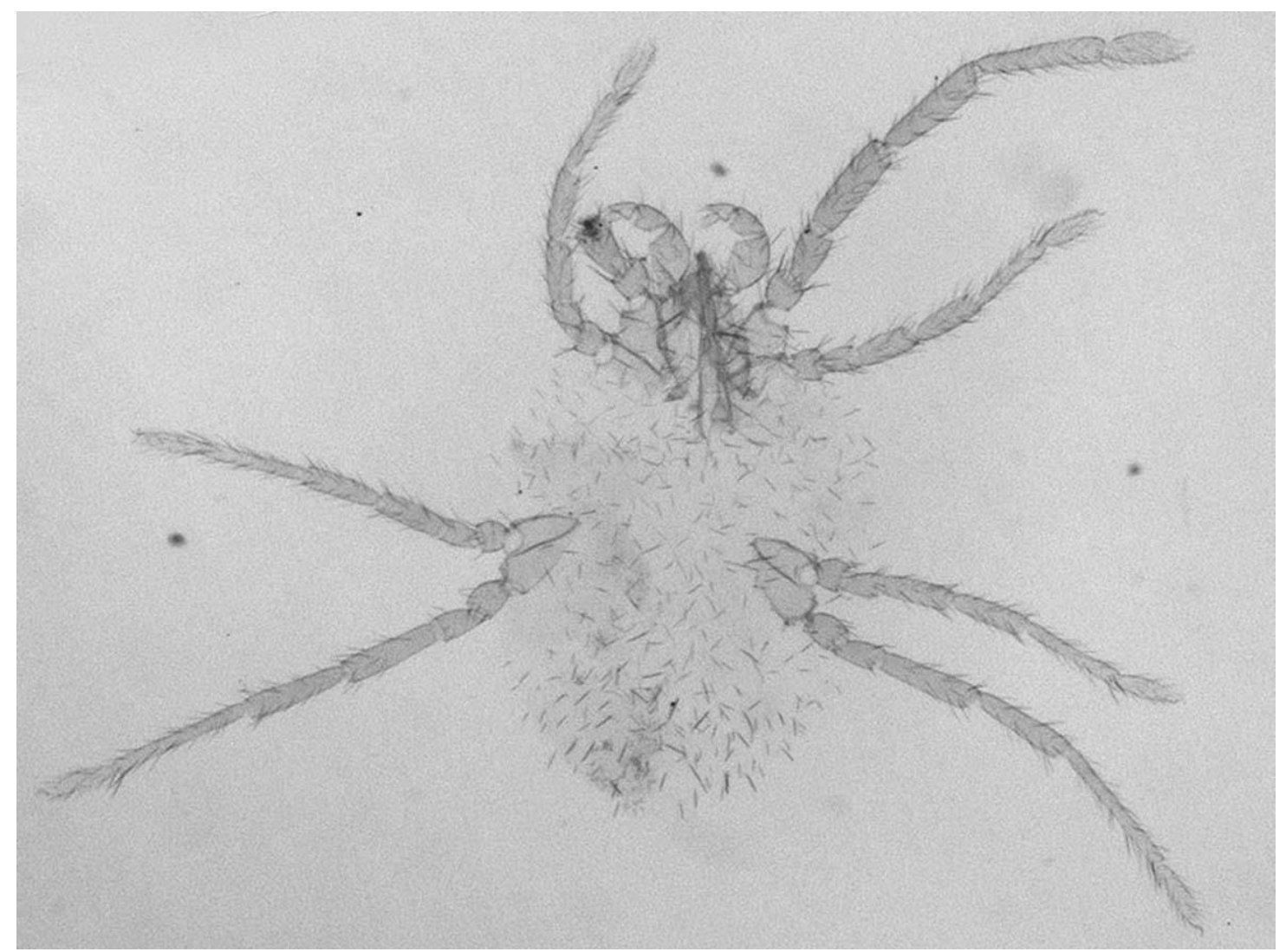

Figure 1. Curteria curticristata (Willmann, 1951) (deutonymph), General view (not to scale)

Description. Deutonymph (Figures 1-7). Body length 970, width 680 (for other measurements see Table 1). Body colour is reddish brown.

Gnathosoma. Chelicerae typical for the genus. Palp trochanter with one smooth seta. Palp femur, genu and tibia with several smooth setae. Tibial claw with a hook placed near its base. Palp tarsus with 5-6 distal eupathidia, 4-5 short, spine-like setae and one solenidium. Palp tibia and genu without conalae (Figure 2). 
Table 1. Compression of morphometric data on active postlarval forms of Curteria curticristata (Willmann, 1951). DN=Deutonymph, $\mathrm{AD}=$ Adult.

\begin{tabular}{|c|c|c|c|}
\hline \multirow[b]{2}{*}{ Characters } & \multirow[b]{2}{*}{$\begin{array}{c}\text { C. curticristata } \\
\text { (DN) } \\
\text { Turkey }\end{array}$} & \multicolumn{2}{|c|}{ from Gabryś (1992) } \\
\hline & & $\begin{array}{c}\text { C. curticristata } \\
\text { (DN?) } \\
\text { Switzerland }\end{array}$ & $\begin{array}{c}\text { C. curticristata } \\
\text { (AD?) } \\
\text { Austria }\end{array}$ \\
\hline LB & 970 & 1032 & $1900-2600$ \\
\hline WB & 680 & 680 & $1500-1700$ \\
\hline ASRo & 170 & - & +100 \\
\hline PSG & 360 & - & - \\
\hline GAn & 130 & - & - \\
\hline GOp & 360 & - & - \\
\hline $\mathrm{AnOp}$ & 190 & 330 & - \\
\hline MDS & $20-38$ & $20-28$ & \pm 27 \\
\hline PDS & $20-50$ & $22-50$ & \pm 27 \\
\hline MVS & $30-36$ & $30-34$ & - \\
\hline PVS & $25-45$ & $30-50$ & - \\
\hline $\mathrm{AL}(\mathrm{n})$ & 6 & $4 ? 6 ?$ & $6 ?$ \\
\hline $\mathrm{AL}$ & $48-60$ & $47-57$ & - \\
\hline ASens & 75 & 81 & - \\
\hline PSens & 96-98 & 91 & - \\
\hline CML & 190 & 145 & $380-390$ \\
\hline PSBp & 7 & 6 & - \\
\hline ISD & 155 & - & - \\
\hline $\mathrm{ASBa}$ & 30 & - & - \\
\hline $\mathrm{SBa}$ & 14 & 14 & - \\
\hline SBp & 15 & 15 & - \\
\hline PSAL & 35 & 45 & - \\
\hline PSAW & 36 & 36 & - \\
\hline CMW & 10 & - & - \\
\hline $\mathrm{O}$ & 27 & - & - \\
\hline $\mathrm{O}-\mathrm{O}$ & 275 & - & - \\
\hline OCM & 135 & - & - \\
\hline OAS & 95 & - & - \\
\hline OPS & 60 & - & - \\
\hline $\operatorname{ExG}(\mathrm{L})$ & 35 & 35 & - \\
\hline $\operatorname{An}(\mathrm{L})$ & 50 & 50 & - \\
\hline \multicolumn{4}{|l|}{ Palps } \\
\hline $\mathrm{PaFe}(\mathrm{W})$ & 75 & 75 & - \\
\hline $\mathrm{PaGe}(\mathrm{W})$ & 45 & 42 & - \\
\hline $\operatorname{PaTi}(\mathrm{W})$ & 30 & 42 & - \\
\hline $\mathrm{PaTa}(\mathrm{L} / \mathrm{W})$ & $40 / 17$ & $48 / 26$ & - \\
\hline $\operatorname{PaTr}(\mathrm{L})$ & 47 & 48 & - \\
\hline $\mathrm{PaFe}(\mathrm{L})$ & 110 & 103 & - \\
\hline $\mathrm{PaGe}(\mathrm{L})$ & 75 & 57 & - \\
\hline PaTi (L) & 50 & 52 & - \\
\hline $\operatorname{PaTiCl}(\mathrm{L})$ & 25 & 22 & - \\
\hline $\mathrm{L}$ (sum) & 307 & 282 & - \\
\hline
\end{tabular}


Table 1 (continued)

\begin{tabular}{|c|c|c|c|}
\hline \multicolumn{4}{|l|}{ I } \\
\hline $\mathrm{Cx}$ & 170 & 190 & - \\
\hline $\mathrm{Tr}$ & 70 & 47 & - \\
\hline $\mathrm{bFe}$ & 120 & 87 & - \\
\hline $\mathrm{tFe}$ & 205 & 174 & - \\
\hline $\mathrm{Ge}$ & 230 & 209 & - \\
\hline $\mathrm{Ti}$ & 250 & 221 & - \\
\hline $\mathrm{Ta}$ & 140 & 134 & - \\
\hline $\mathrm{Ta}(\mathrm{H})$ & 60 & 61 & - \\
\hline $\mathrm{L}(\mathrm{sum})$ & 1185 & 1062 & $1605-2232$ \\
\hline \multicolumn{4}{|l|}{ II } \\
\hline $\mathrm{Cx}$ & 130 & 142 & - \\
\hline $\mathrm{Tr}$ & 50 & 47 & - \\
\hline $\mathrm{bFe}$ & 80 & 63 & - \\
\hline $\mathrm{tFe}$ & 120 & 110 & - \\
\hline $\mathrm{Ge}$ & 140 & 142 & - \\
\hline $\mathrm{Ti}$ & 165 & 156 & - \\
\hline $\mathrm{Ta}$ & 105 & 95 & - \\
\hline $\mathrm{Ta}(\mathrm{H})$ & 40 & 40 & - \\
\hline L(sum) & 790 & 755 & - \\
\hline \multicolumn{4}{|l|}{ III } \\
\hline $\mathrm{Cx}$ & 150 & 134 & - \\
\hline $\operatorname{Tr}$ & 60 & 43 & - \\
\hline $\mathrm{bFe}$ & 80 & 59 & - \\
\hline $\mathrm{tFe}$ & 135 & 126 & - \\
\hline $\mathrm{Ge}$ & 150 & 142 & - \\
\hline $\mathrm{Ti}$ & 200 & 197 & - \\
\hline $\mathrm{Ta}$ & 110 & 99 & - \\
\hline $\mathrm{Ta}(\mathrm{H})$ & 32 & 34 & - \\
\hline $\mathrm{L}(\mathrm{sum})$ & 885 & 800 & - \\
\hline \multicolumn{4}{|l|}{ IV } \\
\hline $\mathrm{Cx}$ & 170 & 166 & - \\
\hline $\operatorname{Tr}$ & 95 & 67 & - \\
\hline $\mathrm{bFe}$ & 95 & 47 & - \\
\hline $\mathrm{tFe}$ & 195 & 182 & - \\
\hline $\mathrm{Ge}$ & 200 & 201 & - \\
\hline $\mathrm{Ti}$ & 280 & 248 & - \\
\hline $\mathrm{Ta}$ & 125 & 114 & - \\
\hline $\mathrm{Ta}(\mathrm{H})$ & 35 & 39 & - \\
\hline L(sum) & 1160 & 1025 & $1655-2573$ \\
\hline IP & 4020 & 3642 & - \\
\hline
\end{tabular}



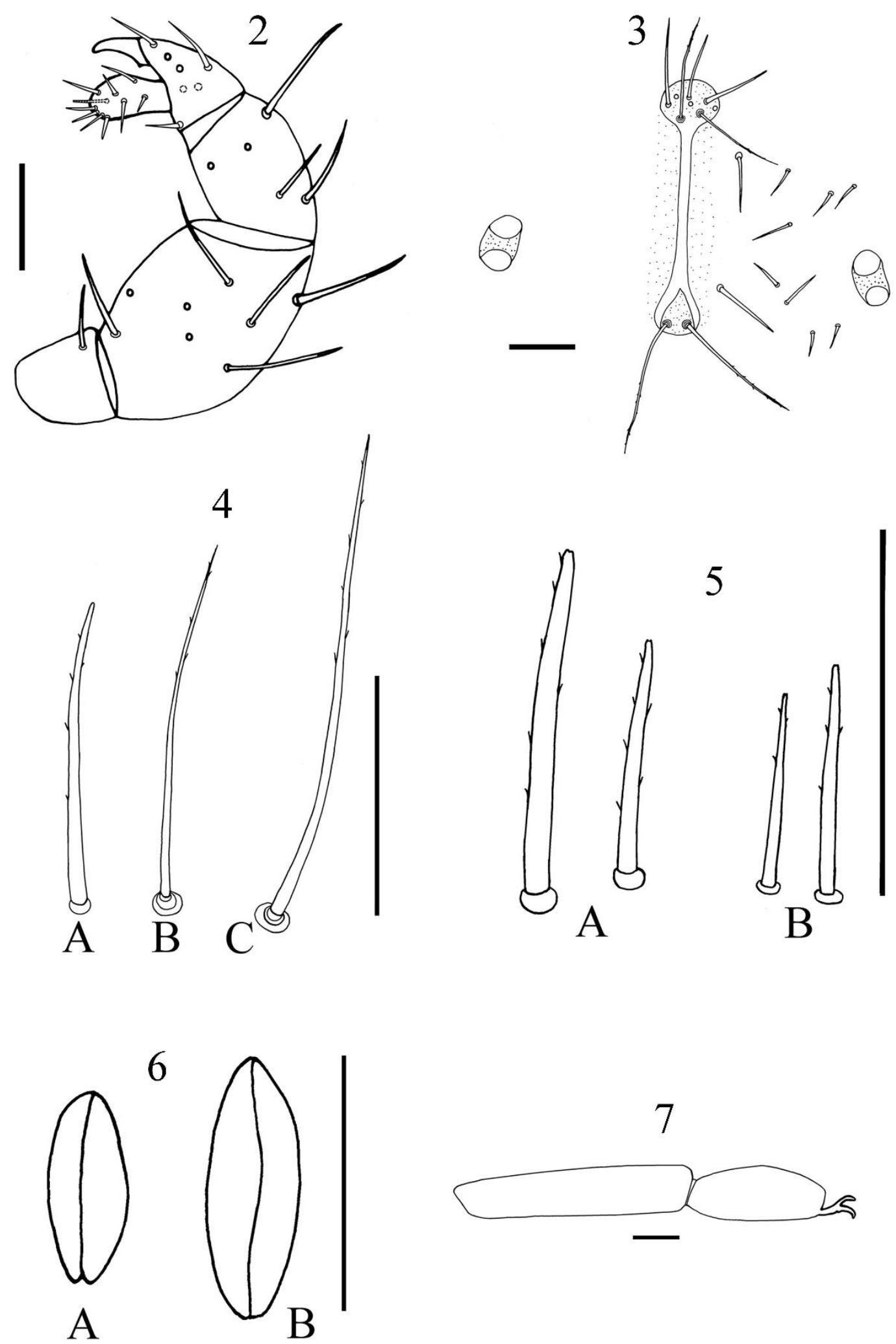

Figures (2 - 7). Curteria curticristata (Willmann, 1951) (deutonymph), 2 Palp, medial view 3 Crista metopica region 4 A) AL seta B) ASens C) PSens 5 A) Dorsal setae B) Ventral setae 6 A) Genital opening B) Anal opening 7 Tibia and tarsus of leg I. Scale bars: $50 \mu \mathrm{m}$. 
Idiosoma. Scutum available. Crista metopica consists of anterior and posterior sensillary areas and a straight rod extending between them. Anterior and posterior processes of crista metopica not detectable. Paired sessile eyes, located at laterally slightly under halfway the length of crista metopica. Posterior lens slightly larger than anterior ones (Figure 3). The anterior sensillary area bears 6 nonsensillary setae with a few barbs (AL) (Figure 4A) and pair sensilla. Anterior sensilla with very short barbs in its distal third (Figure 4B). Posterior sensillary area with a pair sensilla with very short barbs in its distal half (Figure 4C). Posterior sensilla longer than anterior ones. Dorsal setae spiniform, almost smooth, of various lengths (20-50). All dorsal setae originate from small plates. Stems are slightly narrowed terminally and covered sparsely with delicate barbs. Distal end of setal stems blunt shaped (Figure 5A). Ventral setae like dorsal ones but shorter (25-45) and thinner (Figure 5B). Genital opening much shorter (mean 35) while anal opening relatively long (mean 50). Both openings without seta (Figures 6A, B).

Legs. Legs I and IV clearly much longer than legs II-III (legs II shortest). All legs covered smooth or barbed setae. All legs without serratalae. Tibia I longer than tarsus I and length/width of tarsus I $<2.5$ (Figure 7).

\section{Distribution}

Austria, Greece, Switzerland (Gabryś, 1992; Mąkol and Wohltmann, 2012) and Turkey (present study).

Note. Gabryś (1992) approached with suspicion that this species characterized by its "highalpine" was reported by Cooreman (1955) from Greece.

\section{Discussion}

The active postlarval members of Curteria typically have similar dorsal setae (spiniform and almost smooth). The most distinctive feature that distinguishes $C$. curticristata from other active postlarval species of Curteria is its short dorsal setae. The length of dorsal setae is up to 50 in C. curticristata but up to 140 in C. episcopalis, up to 70 in C. fageli, up to 75 in $C$. graeca and up to 85 in C. southcotti (Schweizer, 1951; Willmann, 1951; Gabryś, 1992). The habitat (at an altitude of $2230 \mathrm{~m}$ ) of deutonymph of $C$. curticristata in Turkey, confirms mountain regions (according to Willmann (1951) "high-alpine") living of this species.

\section{Acknowledgements}

I thank Prof. Dr. Grzegorz Gabryś for providing some references. Also, I thank anonymous reviewers for their contributions on the MS. 


\section{Ethics}

This study does not require ethical approval.

\section{References}

Cooreman, J. 1955. "Note sur des Acariens recoltes en Grece et en Turquie par K. Lindberg”, Bulletin et Annales de la Société entomologique de Belgique, 41(5-6), 119-130.

Gabryś, G. 1992. "Curteria southcotti sp. n. from Poland with redescription of C. episcopalis (C. L. Koch, 1837) comb. nov. (Acari: Actinedida: Erythraeidae)", Genus, 3, 243-259.

Mąkol, J. and Wohltmann, A. 2012. "An annotated checklist of terrestrial Parasitengona (Actinotrichida: Prostigmata) of the world, excluding Trombiculidae and Walchiidae", Annales Zoologici, 62, 359-562.

Saboori, A., Çobanoğlu, S. and Bayram, S. 2007. "A new genus and species of larval Erythraeinae (Acari: Erythraeidae) from Turkey", International Journal of Acarology, 33(4), 359-363.

Schweizer, J. 1951. "Die Landmilben des Schweizerischen Nationalparkes", 2 Teil: Trombidiformes Reuter 1909", Ergebnisse der wissenschaftlichen Untersuchung des schweizerischen Nationalparks, III, 23(2), 51-172.

Schweizer, J. and Bader, C. 1963. "Die Landmilben der Schweiz (Mittelland, Jura und Alpen), Trombidiformes Reuter", Mémoires de la Société Helvétique des Sciences Naturelles, 84(2), 209-378 + I-V.

Southcott, R. V. 1961. "Studies on the systematics and biology of the Erythraeoidea (Acarina), with a critical revision of the genera and subfamilies", Australian Journal of Zoology, 9(3), 367-610.

Walter, D.E. and Krantz, G.W. 2009. "In: Krantz G.W. and Walter, D.E. (Eds.), Collecting, rearing, and preparing specimens", A Manual of Acarology. 3rd Edition, Texas Tech University Press, Texas, 83-96.

Willmann, C. 1951. "Die hochalpine Milbenfauna der mittleren Hohen Tauern insbesondere des Großglockner-Gebietes (Acari)", Bonner zoologische Beiträge, 1(2), 141-176.

Wohltmann, A., Mąkol, J. and Gabryś, G. 2007. "Description of the larva of Curteria Southcott, 1961 (Acari: Parasitengona: Erythraeidae) with notes on biology and life cycle", Biologia, 62(5), 573-580 